\title{
Help Me to Understand Your World: A Reflection on the Potential Impact of E-Health Systems on the Prognosis for Asperger Syndrome
}

\author{
Rudi G. Harmse and Dalenca Pottas \\ Faculty of Engineering, the Built Environment and Information Technology, \\ P.O. Box 77000, Nelson Mandela Metropolitan University, PORT ELIZABETH, 6031, \\ South Africa \\ \{Rudi, Dalenca\} @nmmu.ac.za
}

\begin{abstract}
Consumer focused e-Health systems will provide a broader spectrum of health consumers with timely, cost-effective and relevant services that will ultimately lead to a greater quality of life. This is the promise (or hope) that is increasingly being heard from health informatics researchers and practitioners. This paper looks at the characteristics of a particular minority group, namely high-functioning adults with Asperger Syndrome. It considers opportunities and risks associated with the worldwide web that has been identified in the literature and relates its implications for this community. Finally the paper concludes with some reflections on the questions that need to be answered.
\end{abstract}

Keywords: Autism Spectrum Disorder, Asperger Syndrome, e-Therapy, e-Health.

\section{Introduction}

There has been significant development in the understanding of the concept "Autism" over the last 3 decades. This has led to the diagnostic concept being broadened and the realization that previous prognosis expectations need to be re-examined [1].

The various diagnostic concepts are now commonly collectively referred to as Autism Spectrum Disorders (ASD) and reflects the consensus that Autistic Disorder and related disorders should be thought of as a spectrum. Individuals diagnosed with an ASD all demonstrate the same core deficits but the symptoms may manifest in diverse ways. With, for example, the intellectual function ranging from severe mental retardation to IQs in the superior range on conventional IQ tests.

While the increased awareness of ASD has led to a greater availability of support services there are still many reasons to believe that the amount of available support is inadequate and that the capacity of the traditional health care systems is insufficient. Especially in the case of higher functioning individuals they may not receive the benefit of services which could have a substantial impact on their quality of life. This is particularly evident when the situation of those who managed to avoid detection before adulthood is considered as even less services are available later in life. 
E-Health systems utilize information and communication technologies together with e-commerce and e-business principles to change the way health care is delivered [2]. Consumer focused e-health systems aim to empower users to take greater responsibility for their own health and wellbeing through providing relevant and costeffective services at the point of need. In order to do this it is necessary to understand the needs of the consumers, especially when the relevant consumer population is defined by cognitive processes that differ from the norm.

This paper provides a brief outline of Asperger Syndrome (AS) followed by a consideration of the value of interventions in the life of individuals with AS. The advantages and disadvantages that web based systems have, as identified by Tantam [3], are then related to the particular AS characteristics in order to illustrate ways in which individuals with AS may benefit from these technologies, as well as how the characteristics may either mitigate threats or provide additional challenges.

\section{Autism Spectrum Disorder and Asperger Syndrome}

Asperger syndrome (AS) is one of 3 diagnostic categories identified in what is commonly called the Autism Spectrum Disorders (ASD). This is also sometimes referred to as the Autistic Spectrum Disorders. At one stage autism was considered a rare disorder with a prevalence of about $0.04 \%$ with $70-80 \%$ of these having significant learning disability but more recently the prevalence of the extended spectrum of autistic disorder places the figure at $0.6 \%$ with $70-90 \%$ having normal learning ability [4].

The common characteristic that links all the ASD diagnoses is the widely recognized triad of impairments in the areas of communication, social skills and behavioral flexibility. Currently the diagnosis of AS requires that speaking is not delayed and occurs at the normal age and that the level of IQ is not below normal levels.

Cashin and Barker [5] has recently suggested that, while useful for diagnosis, the triad of impairments focused on behavior is less useful for therapy. They proposed a switch to a cognitive triad of impairments that is constant and not changing at various times as is the case with the behavioral triad which is the manifestation of these underlying cognitive impairments. In this view the actual triad is seen to be visual processing, impairments in abstraction and an impaired theory of mind. If this or a similar common cognitive model could be identified and verified it would be useful for modeling and tracking progress through the life of an individual with AS using an e-health system.

Impaired theory of mind has been referred to as Mindblindness. This theory of Mindblindness can effectively explain the social and communication difficulties faced but it cannot explain non-social features, does not account for the emotional response difficulties and is also not specific to ASD as other clinical conditions also show forms of mindblindness [6].

Baron-Cohen [6] explores the educational implications of extending the theory of Mindblindness to be able to explain the non-social areas of strength present in autism and AS. This was done by adding a second factor (systemizing) and by broadening the theory of mind to include emotional response to the feelings of another (affective empathy) [6]. Systemizing refers to the tendency and ability to analyze or construct 
systems. There are many kinds of systems including mechanical, abstract, natural and even social systems that can be analyzed and understood by noting any regularities and underlying rules. There is evidence for intact and even unusually strong systemizing in AS for example in above the norm performance in a physics test [6].

Under this empathizing-systemizing (E-S) theory both the social-communication deficits as well as the areas of strength can be explained. The deficits are explained in terms of delays in the development of empathy and the strengths in terms of superior skill in systemizing [6]. One of the implications highlighted by Baron-Cohen [6] is that the functioning in the weak area of empathizing can be strengthened by framing learning in terms that allows its uptake by the systemizing strength in a similar way as one might teach a foreign language.

Individuals with AS generally have less severe autistic behavior, higher IQs and better language skills than individuals with Autism Disorder. They also show a desire to interact with others but lack the skills to do so successfully. These combined with the fact that AS is more often only diagnosed later in life creates a different set of opportunities and difficulties for potential intervention. In adults the difficulties are often subtle and are especially present in communication, social relationships and interests [4].

\subsection{Diagnosing the Core Syndrome of AS}

The ICD-10 definition of AS includes exclusion criteria, examples of stereotyped and abnormal behavior and descriptions of abnormalities in reciprocal social interaction.

With the definition of AS including an IQ in the normal or superior range together with better verbal skills a more positive prognosis could be expected. This has been confirmed by studies that found that early communication skills and intellectual level are the most significant predictors of outcomes in adults with ASD [7; 8]. The recognition of the much larger group of people with AS who have social functioning at a level sufficient to have avoided being previously diagnosed has also increased understanding of what can be achieved [9].

A difficulty in practice is that the core syndrome will manifest differently in different individuals as well as over time in the same individual $[9 ; 10]$. While present throughout life, the non-verbal difficulties become progressively harder to determine with age as the individual develops various coping strategies that disguise the difficulties.

Another difficulty is that there are psychological disorders which may be confused with AS or conversely AS could be confused for another psychological disorder [9] which could result in inappropriate treatment.

\subsection{Emotional Consequences of AS and Psychological Disorders}

The prevalence of anxiety and depression is high in people with AS. A complicating factor is that many people with AS attend mainstream schools where their special educational needs may not be recognized.

A common response to distress is the development of an anxiety disorder but this is difficult to diagnose as the main presentation of this may be as an apparent increase of 
the severity of the core AS characteristics. This may lead to an increase in rituals or an agitated state with increased bewilderment [9].

Extended periods of anxiety can lead to depression and increased levels of substance abuse. Depression can also lead to an increase in self-neglect and social withdrawal. The role of emotional factors on the prognosis should not be underestimated and should be a particular focus of assistance [9].

\section{Interventions}

Early detection and the provision of specific education has improved prognosis [10]. In addition, the understanding of the role that emotional and personal factors play in prognosis means that focused interventions aimed at these difficulties can lead to substantial improvements in the apparent level of disability in AS.

\subsection{Quality of Life}

The term "Quality of life" refers to a set of factors that together express personal wellbeing. There are eight quality of life domains recognized (emotional wellbeing, interpersonal relationships, material wellbeing, personal development, physical wellbeing, self-determination, social inclusion and rights).

Renty and Roeyers [11] found a relationship between the perceived quality of life of persons with ASD and the support, both perceived informal as well as received formal and informal, that they experience. They found that the various support characteristics had a greater determining factor on quality of life than the disability characteristics themselves. Interestingly their analysis did not show a strong relation with the level of received support (either formal or informal) but that a correlation was found with perceived informal support as well as a negative correlation with unmet formal support needs.

This would indicate that both the perceptions and specific needs of the individual should be addressed in any interventions to improve quality of life. In the case of unmet support needs the following were found to be most correlated: accommodation, daytime activities, ASD-specific information, and interpersonal relationships [11]. Of these it will be seen in section 4 that web-based technologies provide particular opportunities for the last two.

\subsection{Value of Interventions}

When there is clearly a disorder present that is susceptible to medication this could be used, but it has been shown that individuals with AS may be particularly susceptible to the side-effects of medication. They should therefore be started at lower dosage and any increases should be in smaller increments than would normally be used [9].

With the significance of met and unmet formal support needs there should be a particular focus on identifying and meeting these needs. With the findings that targeted formal support is of much more value this would appear to be an area where investments in this regard can be leveraged by ensuring it is targeted and specific. It is clear that what is required is a person-centered methodology that provides individuals with the means to strengthen their social support network as well as support that is 
completely tailored to their needs. Could web-based e-health provide such an environment? What other opportunities and threats does the e-health approach bring with it?

\section{Opportunities and Threats}

Tantam [3] explores a number of opportunities and threats posed by the worldwide web for psychotherapy in an e-health approach under the categories:

- Information

- Interactivity

- Openness

- Disembodied presence

These categories will be used here to frame the discussion. Of these categories, the first two are related to the important support needs that are strongly correlated with quality of life mentioned in section 3.1. That is the ASD-specific information and the interpersonal relationships that are related to the Information and Interactivity categories respectively. The other two categories have not yet emerged as having such a direct bearing on AS prognosis but they were considered for completeness and it will be seen that they have some implications as well.

\subsection{Information}

A particular advantage of the new technologies is the availability of information in its various forms. There are many sources of information available, but with this availability comes the problem of information overload. The quality of information is also of some concern with the dangers of misinformation and disinformation being ever present [3].

Through the simple expedience of a search on the Internet using for example a search engine such as Google a person with AS can gain access to a diverse collection of information but what is the value of this information? With a number of different competing theories as well as unproven treatment methods there is potential for confusion, outdated or even biased information.

If, as occurs frequently, the individual with AS makes learning about their condition a particular interest then their capacity for intense focus and persistence on their special interest becomes an asset in dealing with the amount of information available. It has also been found that for individuals with AS reading is a much more effective method of understanding than listening [4].

Individuals with AS do, however, seem to have some problems with executive control functions which make it harder for them to structure their information search and focusing on the most relevant materials. Many individuals compensate for these weaknesses by imposing strict structure on their lives and planning in detail.

There is also the concern that these individuals' trusting nature and tendency to literal interpretation may make them susceptible to misinformation or lead to misunderstanding some of the material. 
Given the importance of the availability of ASD-specific information any e-health solution targeting this community will need to provide this information with the necessary quality control. Specific attention would need to be paid to ensuring that the material is clear, appropriately structured and not misleading to members of the AS community.

\subsection{Interactivity}

Communication technologies have created greater opportunities for interactivity than ever before. This increased communication can assist in clarifying advice and provide a channel of feedback [3]. It could also assist the healthcare customers in formulating their problems clearly.

These technologies are useful to those with an ASD in two ways in particular.

Firstly, communication technology could provide them an alternative means to communicate. This is particularly the case with those individuals with more severe communication impairments for whom communication through email and discussion forums are their primary form of communication. It is however rare for an individual with AS to be nonverbal and this is more relevant for some Autism Disorder cases unless another condition such as severe social anxiety is also present.

Secondly, the communication technology makes it possible to bring together individuals from a much larger population. Given the relatively low incidence of ASD an individual is not likely to encounter many other individuals with similar experiences without this access to a wider population. The technology could also make contact with support services possible where it might not be locally available. The wider reach could also make it easier for such specialized support services to reach enough people to justify their existence which is an important consideration for the viability of e-health systems [2].

There is a dark side to all this interactivity. This increased interactivity could lead to unreflective responses and there is the ever present danger of breaches of confidentiality if for example emails are redistributed [3]. The problems individuals with AS have with understanding social conventions and a tendency towards excessive openness could make this even more likely.

The lack of needing to worry about nonverbal communication allows the individual with AS to focus on what needs to be said but there is the danger that indirect forms of communication could be used to such an extent that the individual withdraws from other interactions. In this way any social anxiety could be reinforced and lead to excessive withdrawal.

As mentioned earlier, individuals with AS want to socialize. This means that they are likely to make use of the opportunities to interact with peers provided by the technology. There is unfortunately the danger that they may be targeted for abuse as they may be perceived as a "soft target".

A number of websites have been created by people with particular conditions and many of these incorporate some form of discussion forum where a community forms that provides support to each other held together by their common experience. As discussed in section 3.1 the level of perceived informal support has a bearing on perceived quality of life. Some of this informal support may be obtained through online support groups. 
Anecdotal evidence is mixed as to the helpfulness of this support and the general lack of systematic evidence suggests that professionals need to become more involved in the provision of information and support as well in the evaluation thereof [12].

Some examples of such online support groups for ASD include:

www.wrongplanet.net

http://www.grasp.org/

http://www.aspergersyndrome.org/

Given the demonstrated value of intervention, having an increased access to relevant services is likely to be an advantage. There is however currently still the questions surrounding the quality of e-services with the susceptibility to medicine side effects in the AS population being of concern as well as some inconsistency in diagnosis. This is a factor that is not only relevant for systems specially designed for AS but is particularly of concern in more general e-health systems where a misdiagnosis of for example Schizophrenia could lead to the prescription of medication which will result in a poor prognosis for the individual with AS.

The potential for gaining access to specialists needs to be balanced with the practical problems and limitations. On the one hand the fact that so much of psychological intervention is based on communication makes it suitable with a virtual environment - certainly much more so than medical interventions requiring a physical presence. On the other hand the interaction is not as easily reduced to simple data items as would be the case in remote monitoring of simpler physical ailments such as blood sugar levels in patients with diabetes. The therapist-client relationship could also arguably be more dependent on the establishment of a trust relationship for success which may be more difficult to establish in the disembodied environment.

\subsection{Openness}

Very little information on the Internet can be restricted to only professionals [3]. This openness allows health consumers to view much of the same information as professionals, interpret this information themselves and even participate in professional disagreements [3].

Given the still volatile nature of our understanding of AS and the amount of ongoing research there is the danger that consumers might be exposed to findings and conclusions that may in fact be premature. The many different views and academic debates may also lead to confusion among consumers.

Individuals with AS may be prone to developing their own theories based on this information due to their extreme tendency to systemizing. Obviously this poses a danger, but at the same time if those individuals with high cognitive abilities could apply their abilities in conjunction with experts within the field it could lead to furthering our understanding of the condition.

\subsection{Disembodied Presence}

The risks so far mentioned are not unique to the Internet and have been present in various degrees in other media. A particular aspect where the digital media does pose a new challenge is in the aspect of disembodied presence [3]. 
The issue here is the unprecedented degree of anonymity and the ease of deception of even such fundamental characteristics as gender and age [3].

There is also a lack of presence that being with a person normally entails. Even when there is no verbal communication there is still the constant stream of non-verbal communication [3]. It may be possible to create a sense of presence through a computer conversation, but once again the reality that all is not what it seems due to the possibility of virtual identities is present.

Given the fact that one of the defining characteristics of ASD is a reduction in awareness of non-verbal communication it might be expected that those with ASD would be less affected by this aspect of disembodiment in the same way that a blind person is unaffected by a darkened room while it has a major impact on sighted individuals. There is still the reality that all is not as it seems with virtual identities and as individuals with ASD are seen as socially naive they may well become the targets of purposeful deception.

Tantam [3] explores the concept of embodiment further and also considers the effect that the absence of a physical awareness of the presence has on the conduct of individuals online. While a computer with a camera can have a field of vision, there is no actual gaze that could generate a sense of shame. This can be an advantage in situations such as computerized tests or to allow individuals unable to bear public scrutiny to interact but can also lead to behavior that would normally be inhibited due to the moderating effect of shame [3].

Interestingly some of the socially problematic behaviours of individuals with ASD may stem from a reduced awareness of others. It is possible that such individuals may therefore also be expected to experience less of a difference in this regard. At the same time there is the risk that an excessive amount of time spent in virtual communication could further reduce opportunities for the individual to develop an increased awareness of the presence of others and to modulate behavior appropriately.

There can also be a degree of dissociation with the Internet serving as an alternative world [3]. Given the high incidence of anxiety and depression in individuals with AS, there is always the danger that such individuals may withdraw into the virtual world. They could then use it as a method to avoid facing the difficulties of real world interactions. If this avoidance gives them access to more interaction it is a positive aspect but a reduction of real world interaction should be guarded against.

\section{Summary and Discussion}

Table 1 provides a summary of the information presented in this paper with the AS characteristics that have relevance for the opportunities and threats discussed. In this table those characteristics that could be considered as positive in the particular context is indicated with $\mathrm{a}+$ while those that provide a particular challenge to an individual with AS is indicated with a -.

As can be seen in the table AS provides a number of problems in all these areas but also present a number of particular advantages for each of the categories considered. It would therefore seem reasonable to conclude that there is reason to believe that it 
Table 1. Summary of opportunity and threats related to relevant AS characteristics

\begin{tabular}{|c|c|c|c|}
\hline Category & Opportunity & Threat & AS characteristics \\
\hline Information & Availability & $\begin{array}{l}\text { Information overload } \\
\text { Mis-/Disinformation }\end{array}$ & $\begin{array}{l}\text { +Intense focus } \\
+ \text { Persistence } \\
\text {-Executive control issues } \\
\text {-Relevance of focus } \\
\text {-Literal interpretation } \\
\text {-Trusting }\end{array}$ \\
\hline Interactivity & $\begin{array}{l}\text { Clarifying advice } \\
\text { Alternative } \\
\text { communication } \\
\text { Links with peers } \\
\text { Access to services }\end{array}$ & $\begin{array}{l}\text { Unreflective } \\
\text { responses } \\
\text { Confidentiality } \\
\text { breach } \\
\text { Withdrawal } \\
\text { Abuse } \\
\text { Variable quality }\end{array}$ & $\begin{array}{l}\text {-Social conventions } \\
\text {-Excessive openness } \\
\text { +Relative verbal strength } \\
\text {-Social anxiety } \\
\text {-Prone to victimization } \\
\text { +Desire to socialize } \\
\text {-Inconsistency in diagnosis } \\
\text {-Susceptible to side-effects } \\
\text { +Value of intervention }\end{array}$ \\
\hline Openness & $\begin{array}{l}\text { Availability } \\
\text { Participation }\end{array}$ & $\begin{array}{l}\text { Premature } \\
\text { information }\end{array}$ & $\begin{array}{l}\text {-Develop own theories } \\
+ \text { Systemizing ability }\end{array}$ \\
\hline $\begin{array}{l}\text { Disembodied } \\
\text { presence }\end{array}$ & Anonymity & $\begin{array}{l}\text { Withdrawal } \\
\text { Deception }\end{array}$ & $\begin{array}{l}\text { +Nonverbal deficits } \\
\text {-Behaviour reinforcement } \\
\text {-Socially naïve }\end{array}$ \\
\hline
\end{tabular}

would be fruitful to utilize these technologies for this population but that the solutions need to be tailored to their particular difficulties to mitigate for the particular weaknesses. There are a number of ways where these technologies are already being utilized. One that has led to an increase in the number of people who realize they may have AS is online testing.

Using the Internet for testing has become very popular as it is easy to do with the current technology and has been shown to give results that are similar to those administered by a human [13]. The capabilities of computers to conduct analysis of complex tests that would be time consuming by hand as well as the ease with which tests can be converted for Internet use means that tests that are selected, administered and rated by users have become widely available. This proliferation of selfadministered tests has to be balanced with questions of validity. Just because a clinical test has been converted to an Internet form does not mean that its results have the same validity. The validity of a self-administered test has to be tested, even if it is based on a test that has proven validity when conducted by another person the selfadministered nature could result in effectively being a different test.

Tantam [13] believes that the main advantage of computer-based testing is the fact that these tests can be adaptive and therefore able to ask only the most discriminating questions to achieve a specific level of accuracy with only the necessary questions being asked. The efficiency of testing means that repeat testing can be used to track progress. It would be important to ensure that the tests maintain their validity and 
reliability after extended use and that they do not simply measure improved skill at taking the test.

An interesting finding is that, when the content could be considered shameful, computer-based tests are answered more honestly than human administered tests. So, at least in some instances, these tests could have even more validity than human administered tests.

There is the risk that individuals might take online tests without a sufficient understanding of their significance or shortcomings. A common type of message seen on online AS forums is: "I took testXYZ online and got a score of X. What now? What does that mean?" Easily available screening tests may lead to individuals seeking appropriate help which they might not have realized they need otherwise. An individual with an undiagnosed ASD might be functioning sufficiently to have escaped diagnoses and may never have been formally tested. Yet they have experienced difficulties throughout their lives and been aware of being "different". In many cases an online test provides the first step towards an improved understanding of their own mental functioning and allows them to come in contact with others that think similarly and ultimately lead to a better quality of life.

The benefit for people who would not otherwise have become aware of the origin of their problems should be balanced with the risks that people might inappropriately self-diagnose on the basis of online screening tests without understanding the limitations and purpose of the tests.

Another area where it might be possible to expand the support available is if certain services could be automated. There are some areas of psychotherapy that utilize well tried procedures which, at least to some extent, could be implemented in algorithms that a computer could follow. Tantam [13] refers to a few studies that concluded that computer based treatment could be a viable option for psychotherapy aimed at the reduction of distress.

Given the high level of incidence of anxiety and the importance of emotional and personal factors for the prognosis of individuals with AS (as discussed in section 3), it is clear that if effective computerized systems were available to assist with this, it would be of help for this community. The difference in cognitive functioning of AS individuals would need to be considered and the validity and effectiveness of these techniques for AS individuals cannot be assumed.

An area for computer therapy that has seen increasing use is the use of immersive virtual environments for various uses [13]. Some relevant uses reported include the fear and avoidance of public speaking as well as autism [13]. Besides the suitability of virtual environments for exposure therapy it has also been used for training such as for social skills training for people with AS.

\section{Reflections}

The use of web based e-health systems hold considerable promise but the particular characteristics of the AS community raises the possibility that response to these technologies may not follow a pattern exactly like those of the general population. It is therefore necessary to conduct further research to determine in what ways these differences impact on the way services are delivered. 
A number of questions still need to be answered or require more definitive answers:

- Given the prevalence of conditions such as anxiety and depression, does the effectiveness of treatments for these differ for this population?

- Which aspects of support can be automated and safely provided? What is the long term effect of such support?

- Given the prevalence of usage of Internet based testing by consumers and the value of early detection and educational support, how can e-health systems support this process while minimizing misuse?

- Given the mixed experiences with peer support, what factors influence its success?

- How can professional and peer support more closely integrate to leverage the advantage of peer support while mitigating for its risk factors?

- To what extent does a sufficient level of trust develop in the therapist-client relationship in an e-health solution? Does the AS characteristics make this more or less likely to occur?

- The understanding of AS is continually evolving and studies of long-term prognosis is by its nature based on treatments conducted in the past. How can e-health systems assist in assessing the effectiveness of newer treatments?

- How can e-health systems utilize the systemizing strengths to assist the consumers to overcome their tendency for trusting inappropriately and being too open with self-disclosure?

\section{Conclusion}

This paper considered the characteristics of a particular group of potential health consumers, namely those with Asperger Syndrome (AS). It was concluded that a person-centered methodology that provides individuals with the means to strengthen their social support network and received support that is completely tailored to their needs is desired. The question was then asked: Could e-health provide such an environment?

The particular characteristics of individuals with AS were then considered in terms of the opportunities and threats that can be identified in the categories: Information, Interactivity, Openness and Disembodied presence.

This led to the conclusion that there are potential benefits and that some types of systems have already been implemented but it was also seen that this population brings a number of special considerations that need to be given attention. It cannot be assumed that their response to these systems will be the same as the general population and that the effectiveness of approaches should be verified for this population. This includes ensuring that systems aimed at the general population which include individuals with AS but are not specifically focused on their particular needs at least "above all - do no harm". 


\section{References}

1. Marriage, S., Wolverton, A., Marriage, K.: Autism Spectrum Disorder Grown Up: A Chart Review of Adult Functioning. J. Can. Acad. Child Adolsc. Psychiatry 18(4), 322-328 (2009)

2. Tan, J.: E-Health Care Information Systems: An Introduction for Students and Professionals. Jossey-Bass, San Francisco (2005)

3. Tantam, D.: Opportunities and Risks in e-Therapy. Advances in Psychiatric treatment 12, 368-374 (2006)

4. Berney, T.: Asperger Syndrome from Childhood into Adulthood. Advances in Psychiatric treatment 10, 341-351 (2004)

5. Cashin, A., Barker, P.: The Triad of Impairment in Autism Revisited. Journal of Child and Adolescent Psychiatric Nursing 22(4), 189-193 (2009)

6. Baron-Cohen, S.: The Empathising-Systemising Theory of Autism: Implications for Education. Tizard Learning Disability Review 14(2), 4-13 (2009)

7. Howlin, P.: Outcome in Adult Life for more Able Individuals with Autism or Asperger Syndrome. Autism 4, 63-83 (2000) doi:10.1177/1362361300004001005

8. Howlin, P., Goode, S., Hutton, J., Rutter, M.: Adult Outcome for Children with Autism. J. Child Psychol. Psychiatry 45(2), 212-229 (2004)

9. Tantam, D.: Psychological Disorder in Adolescents and Adults with Asperger Syndrome. Autism 4, 47-62 (2000) doi:10.1177/1362361300004001004

10. Attwood, T.: The Complete Guide to Asperger's Syndrome (Softcover first edition). Jessica Kingsley Publishers, London (2008)

11. Renty, J.O., Roeyers, H.: Quality of Life in High-functioning Adults with Autism Spectrum Disorder: The Predictive Value of Disability and Support Characteristics. Autism 10, 511-524 (2006) doi:10.1177/1362361306066604

12. Tantam, D.: The Machine as Intermediary: Personal Communication via a Machine. Advances in Psychiatric treatment 12, 427-431 (2006)

13. Tantam, D.: The Machine as Psychotherapist: Impersonal Communication with a Machine. Advances in Psychiatric treatment 12, 416-426 (2006) 